Proceeding Series of the Brazilian Society of Computational and Applied ${ }^{1}$ Mathematics

\title{
Análise Dinâmica de uma Viga de Euler-Bernoulli Submetida a Impacto no Centro após Queda Livre Através do Método de Diferenças Finitas
}

\author{
Bruno Conti Franco \\ Grupo de Mecânica Aplicada, Programa de Pós Graduação em Engenharia, \\ Universidade Federal do Pampa - UNIPAMPA, Alegrete, RS. \\ Wang Chong ${ }^{2}$ \\ Grupo de Mecânica Aplicada, Programa de Pós Graduação em Engenharia, \\ Universidade Federal do Pampa - UNIPAMPA, Alegrete, RS.
}

\begin{abstract}
Resumo. O presente trabalho apresenta análise dinâmica do movimento transversal de uma barra, submetida a impacto provocado por queda livre, pelo método de diferenças finitas (MDF). Os resultados do algoritmo desenvolvido são comparados aos do método de elementos finitos, confirmando que é correto e confiável.
\end{abstract}

Palavras-chave. Análise dinâmica, Viga de Euler-Bernoulli, Método de Diferenças Finitas, ANSYS - LS, Elementos Finitos.

\section{Introdução}

O Brasil é hoje um dos maiores produtores de minério de ferro do mundo. A extração é realizada em diversas etapas, as principais são a lavra, a britagem e a moagem. A moagem é a operação de fragmentação fina, após essa etapa o minério está pronto para ser utilizado em outros processos industriais. $\mathrm{O}$ equipamento mais comum nesse processo é o moinho de Barras, nele a moagem ocorre pelo impacto entre as barras de aço (Figura 1) e as pedras de minério. Essas máquinas apresentam elevado custo de manutenção devido a constante quebra das barras internas devido aos esforços causados pelo choque com as pedras de minério.

Para reduzir o custo de manutenção do equipamento é necessário análise matemática de mecânica da fratura na barra. O impacto entre as pedras e as barras dificulta a modelagem matemática, por que a propagação de trinca induzida por impacto é complexa e difícil de ser modelada matematicamente.

\footnotetext{
${ }^{1}$ bruno.franco@ibiruba.ifrs.edu.br

2 wangchong@unipampa.edu.br
} 


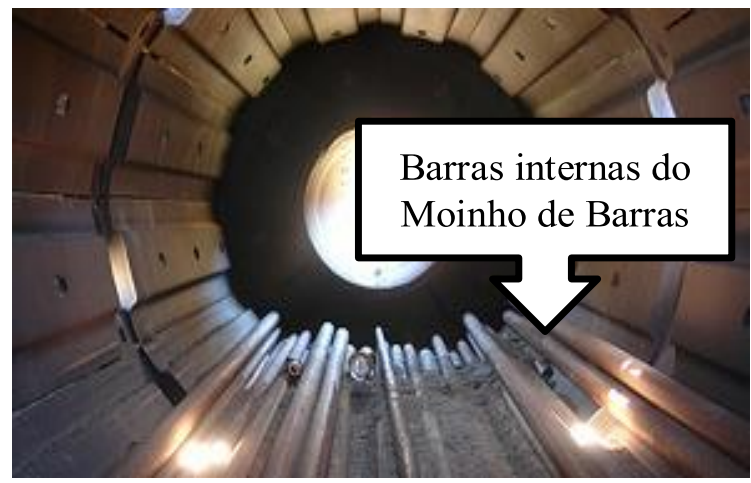

Figura 1: Unidade de processamento de um moinho de Barras

O movimento transversal de vigas tem sido amplamente estudado com vários trabalhos publicados sobre esse assunto. Em estudo apresentado em [1] sobre o efeito de uma trinca na estabilidade dinâmica de uma viga de Timoshenko com extremidades livre submetida a uma força constante ou intermitente, as frequências naturais e os módulos de vibração transversal foram obtidos pelos métodos de elementos finitos e das escalas múltiplas. Na mesma linha, em [2] foi desenvolvida uma técnica numérica para análise de vibrações de vigas de seção transversal circular contendo trincas, porém também é utilizado o método de elementos finitos.

O método numérico mais usado para o estudo da propagação dinâmica de trinca é o Método de Elementos Finitos (MEF). Atualmente os principais softwares comerciais como ANSYS e ABAQUES apresentam custo elevado e exigem alto nível de treinamento para os usuários, e ainda não são capazes de analisar propagação dinâmica de trinca causada por impacto. Por outro lado o MDF é um método numérico simples de fácil aplicação e muito utilizado em pesquisas científicas.

O objetivo deste trabalho é desenvolver um algoritmo de diferenças finitas para modelar a vibração de uma barra do moinho de barras submetida a impacto. Esse algoritmo deve ser aplicado para análise da propagação dinâmica de trinca em trabalhos futuros.

\section{Fundamentação matemática}

\subsection{Equação governante}

Considera-se uma viga uniforme que choca ao centro dela com um suporte rígido após queda livre. A teoria clássica de Euler-Bernoulli ou da flexão pura considera vigas prismáticas uniformes (de seção transversal constante) com comprimento longitudinal como dimensão predominante. Nesse modelo não é considerado a deformação de cisalhamento presente nas seções transversais. Para essas vigas o interesse de estudo são as ações de movimento chamadas de ações de flexão, conforme mostra Figura 2. A equação geral para a vibração lateral forçada de uma viga uniforme [3], é dada por:

$$
\frac{\partial^{2} M(\varsigma, t)}{L^{2} \partial \varsigma^{2}}+\rho A \frac{\partial^{2} w(\varsigma, t)}{\partial t^{2}}=f(\varsigma, t) \quad \text { com } \quad \varsigma=\frac{x}{L}, 0 \leq \zeta \leq 1
$$


onde $M$ é o momento fletor, $L$ é a metade do comprimento total da barra (devido a ${ }^{3}$ simetria), $t$ é o tempo, $\rho$ é a densidade do material, $A$ é a área da seção transversal, $w$ é o deslocamento transversal, $f(\varsigma, t)$ é a força corporal externa $[\mathrm{N} / \mathrm{m}]$ e $x$ é a posição no eixo longitudinal adimensionalizado por $\varsigma$.

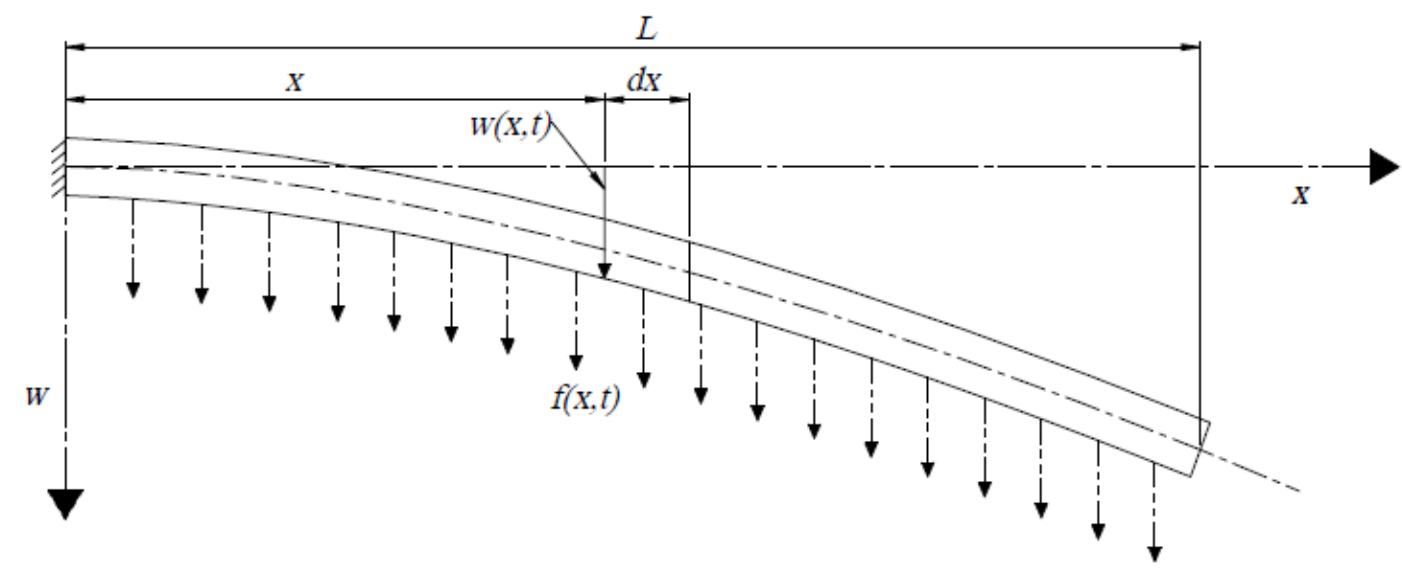

Figura 2: Flexão transversal

A viga é discretizada uniformemente em $n+1$ pontos, a distância entre cada ponto é chamado de passo $h=1 / n$, um valor adimensional, representado na Figura 3.

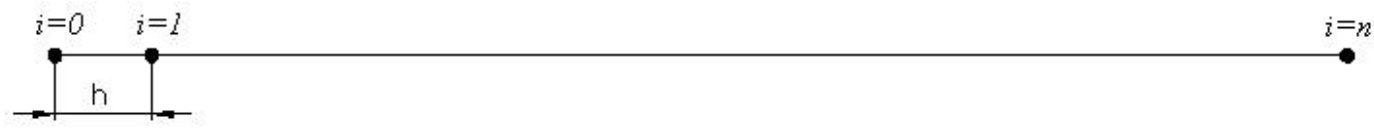

Figura 3: Malha

A expressão $\partial^{2} M / L^{2} \partial \varsigma^{2}$ na Equação (1), corresponde à segunda derivada de $M$ sobre $x$, pode ser representada pela fórmula de diferenças finitas centrada:

$$
\begin{gathered}
\frac{\partial^{2} M_{i}^{j}}{L^{2} \partial \varsigma^{2}}=E I \frac{\partial^{4} w_{i}^{j}}{L^{4} \partial \varsigma^{4}}=\frac{E}{L^{4} h^{4}}\left(I_{i+1}^{j}\left[w_{i+2}^{j}-2 w_{i+1}^{j}+w_{i}^{j}\right]-2 I_{i}^{j}\left[w_{i+1}^{j}-2 w_{i}^{j}+w_{i-1}^{j}\right]+\quad \text { com } 1 \leq i \leq n \text { e } 1 \leq j \leq m\right. \\
\left.+I_{i-1}^{j}\left[w_{i}^{j}-2 w_{i-1}^{j}+w_{i-2}^{j}\right]\right)
\end{gathered}
$$

Onde $I$ é o momento de inércia, $m$ o número de incrementos temporal e utilizando-se da seguinte relação:

$$
M(x, t)=E I \frac{\partial^{2} w(x, t)}{\partial x^{2}}
$$

Ou seja 


$$
M_{i}^{j}=M\left(\varsigma_{i}, t^{j}\right)=E I \frac{\partial^{2} w\left(\varsigma_{i}, t^{j}\right)}{L^{2} \partial \varsigma^{2}}=\frac{E I}{L^{2} h^{2}}\left(w_{i+1}^{j}-2 w_{i}^{j}+w_{i-1}^{j}\right)
$$

A expressão $\partial^{2} w(\varsigma L, t) / \partial t^{2}$ corresponde à aceleração. Para calculá-la utilizamos a formula de movimento linear de um ponto material considerando aceleração como constante com incremento temporal muito curto: $\Delta t=t^{j}-t^{j-1}, j=1,2, \ldots, m$. Assim, pela relação

$$
\Delta s=v \Delta t+\frac{1}{2} a \Delta t^{2}
$$

Obtêm-se:

$$
a=\frac{2(\Delta \mathrm{s}-v \Delta t)}{\Delta t^{2}}
$$

Na forma dicretizada:

$$
a_{i}^{j-1}=\frac{2\left(\Delta \mathrm{s}_{i}-v_{i}^{j-1} \Delta t\right)}{\Delta t^{2}}
$$

Substituindo $\Delta s_{i}=w_{i}^{j}-w_{i}^{j-1}$, então, temos:

$$
\rho A \frac{\partial^{2} w\left(\varsigma_{i}, t^{j}\right)}{\partial t^{2}}=\frac{2 \rho A}{\Delta t^{2}}\left(w_{i}^{j}-w_{i}^{j-1}-v_{i}^{j-1} \Delta t\right)
$$

Substituindo as Equações (1) e (8) na Equação (1) e $f(\varsigma, t)=\rho \mathrm{Ag}$, temos:

$$
\begin{aligned}
\frac{E}{L^{4} h^{4}}\left(I_{i+1}^{j}\left[w_{i+2}^{j}-2 w_{i+1}^{j}+w_{i}^{j}\right]\right. & \left.-2 I_{i}^{j}\left[w_{i+1}^{j}-2 w_{i}^{j}+w_{i-1}^{j}\right]+I_{i-1}^{j}\left[w_{i}^{j}-2 w_{i-1}^{j}+w_{i-2}^{j}\right]\right)+ \\
+ & \frac{2 \rho A}{\Delta t^{2}}\left(w_{i}^{j}-w_{i}^{j-1}-v_{i}^{j-1} \Delta t\right)=\rho A g
\end{aligned}
$$

Após obter $w_{i}^{j}$, pode-se calcular $a_{i}^{j-1}$ pela Equação (7) e em seguida:

$$
v_{i}^{j}=v_{i}^{j-1}+a_{i}^{j-1} \Delta t
$$

\subsection{Condições Iniciais}

A primeira condição inicial é dada por $w(x, 0)=w(\varsigma, 0)=0$, assim temos $w_{i}^{0}=0$. A outra condição inicial é a velocidade inicial da barra no momento em que toca o minério de ferro, dada por quada livre de altura $H$ :

$$
v_{i}^{0}=\sqrt{2 g H}
$$




\subsection{Condições de contorno}

Conforme a Figura 2, a barra encontra-se com uma extremidade engastada e a outra livre assim tem duas condições de contorno em cada extremidade.

Na extremidade engastada $(x=\varsigma L=0$ ou $i=0)$, a deflexão é $w\left(0, t^{j}\right)=0$ e a inclinação é dada por $\partial w\left(0, t^{j}\right) / \partial x=\left(w_{1}^{j}-w_{-1}^{j}\right) / 2 h=0$, então, as condições de contorno para deflexão e inclinação são respectivamente:

$$
w_{0}^{j}=0
$$

e

$$
w_{-1}^{j}=w_{1}^{j}
$$

Na extremidade livre $(\varsigma=1$ ou $i=n)$, temos o momento: $E I(1) \frac{\partial^{2} w\left(1, t^{j}\right)}{L^{2} \partial \varsigma^{2}}=0$. Isto é pela Equação (4):

$$
w_{n+1}^{j}=-w_{n-1}^{j}+2 w_{n}^{j}
$$

Pela força cortante: $\frac{\partial}{L \partial \varsigma}\left(E I(1) \frac{\partial^{2} w\left(1, t^{j}\right)}{L^{2} \partial \varsigma^{2}}\right)=0$, temos:

$$
w_{n+2}^{j}=w_{n-2}^{j}-4 w_{n-1}^{j}+4 w_{n}^{j}
$$

\subsection{Matriz dos coeficientes de deslocamento}

A resolução das equações do sistema linear para o cálculo dos coeficientes de deslocamentos é realizada através das matrizes:

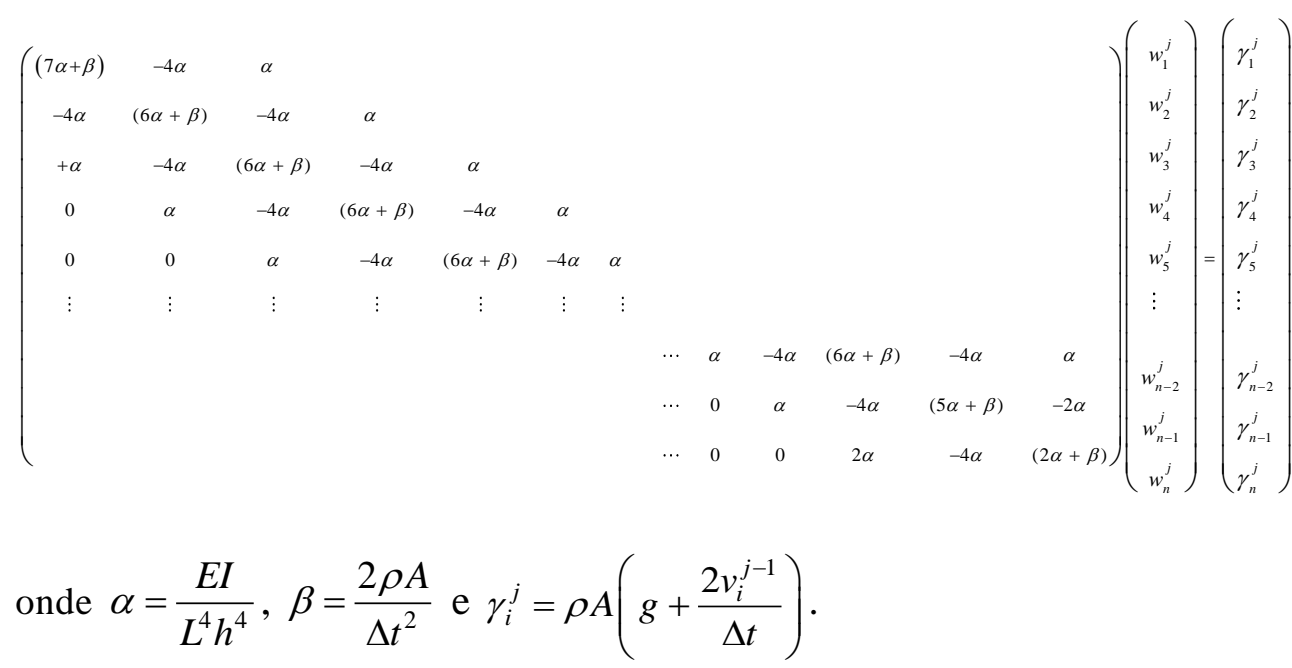




\section{Resultados e discussões}

Os dados de entrada estão listados na Tabela 1.

Tabela 1 - Dados de entrada para as simulações

\begin{tabular}{|l|c|}
\hline \multicolumn{1}{|c|}{ Parâmetro } & Valor \\
\hline Módulo de Elasticidade $(\mathrm{E})$ & $180 \mathrm{GPa}$ \\
\hline Raio $(\mathrm{R})$ & $0.0508 \mathrm{~m}$ \\
\hline Comprimento da metade da barra $(\mathrm{L})$ & $2.21 \mathrm{~m}$ \\
\hline Densidade $(\rho)$ & $7850 \mathrm{Kg} / \mathrm{m}^{3}$ \\
\hline Altura da queda $(\mathrm{H})$ & $6.096 \mathrm{~m}$ \\
\hline Intervalo de tempo $(\Delta t)$ & $1 \mu \mathrm{s}$ \\
\hline Número de discretização $(\mathrm{n})$ & 221 \\
\hline
\end{tabular}

A simulação foi realizada pelo Matlab. Para comparação, usou-se o software ANSYS Mechanical APDL 12.0.1, no módulo LS-DYNA. O elemento selecionado foi o BEAM161, configurado para seção transversal circular.

A figura 4 mostra a comparação dos resultados do deslocamento transversal do último nó da barra, $\mathrm{i}=\mathrm{n}$. Os resultados obtidos pelo MDF e pelo ANSYS são quase idênticos. Isso confirma que o algoritmo desenvolvido é confiável.

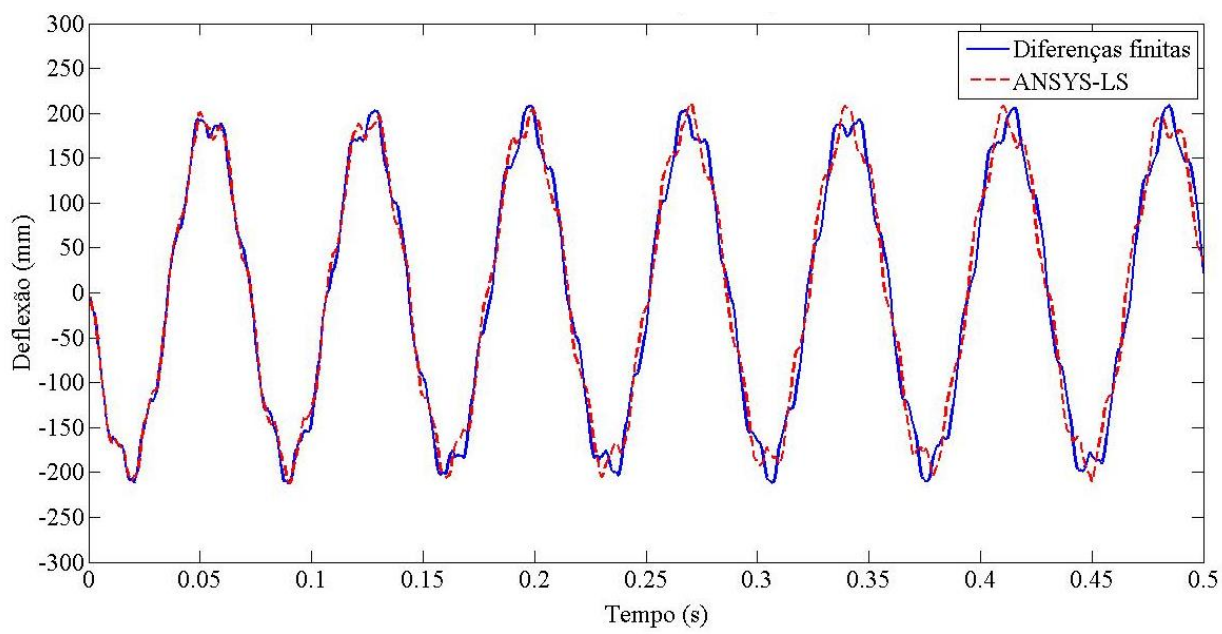

Figura 4 - Deslocamento no último nó da barra $\mathrm{i}=\mathrm{n}$, tempo de 0 a $0.5 \mathrm{~s}$.

A Figura 5 apresenta o momento fletor máximo na barra e o ponto em que ele ocorre. O pico entre 1700 e $1800 \mu$ s pode ser interpretado que existem dois locais (a $8 \%$ e a $50 \%$ do comprimento) na viga onde os momentos fletores são quase idênticos e máximos no instante $1707 \mu$ s, ou seja, as tensões máximas poderão ocorrer nestes dois lugares no mesmo instante de tempo. Isto implica que a barra poderá ser quebrada nestes lugares. 


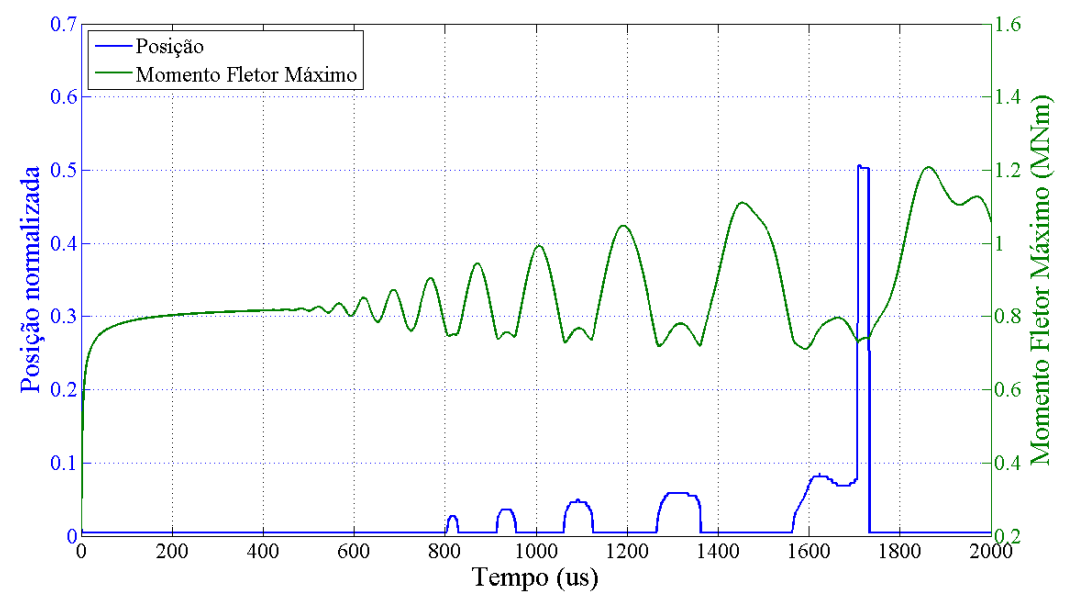

Figura 5 - Posição de Momento Máximo vs Tempo.

\section{Conclusão}

A análise dinâmica do efeito de choque numa estrutura em geral é complexa. O desenvolvimento do programa baseado no MEF para esse fim requer alto nível de conhecimento em MEF. O uso de software comercial pode custar caro além de requer intenso treinamento. $\mathrm{O}$ algoritmo desenvolvido baseado no MDF para análise da viga de Euler-Bernoulli é eficiente e confiável, mas relativamente simples em relação ao MEF. Pelo programa desenvolvido, pode-se obter os perfis de deslocamento, velocidade e aceleração da viga em qualquer instante e em qualquer ponto da viga. Analisando os resultados obtidos, é possível perceber mais de um local na viga onde poderá ocorrer mesma tensão máxima no mesmo instante de tempo. Isso revela que a barra pode ser quebrada em pedaços. O algoritmo desenvolvido poderá ser utilizado em trabalhos futuros para análise de propagação de trinca provocado por choque.

\section{Referências}

[1] K. H. Kim and J. H. Kim, Effect of a crack on the dynamic stability of a free-free beam subjected to a follower force, Journal of Sound and Vibration, vol 1, 119-135, (2000).

[2] M. Kisa and M. A. Gurel, Free vibration analysis of uniform and stepped cracked beams with circular cross sections, International Journal of Engineering Science, vol 1, 364380, (2007).

[3] S. S. RAO, Mechanical Vibrations, Pearson Prentice Hall, vol. 5, (2011). 